\title{
SHARP CONSTANTS OF APPROXIMATION THEORY. I. MULTIVARIATE BERNSTEIN-NIKOLSKII TYPE INEQUALITIES
}

\author{
MICHAEL I. GANZBURG
}

\begin{abstract}
Given a centrally symmetric convex body $V \subset \mathbb{R}^{m}$, let $\mathcal{T}_{a V}$ be the set of all trigonometric polynomials with the spectrum in $a V, a>0$, and let $B_{V}$ be the set of all entire functions of exponential type with the spectrum in $V$. We discuss limit relations between the sharp constants in the multivariate Bernstein-Nikolskii inequalities defined by

$$
\begin{aligned}
& P_{p, q, D_{N}, a, V}:=a^{-N-m / p+m / q} \sup _{T \in \mathcal{T}_{a V} \backslash\{0\}} \frac{\left\|D_{N}(T)\right\|_{L_{q}\left(Q_{\pi}\right)}}{\|T\|_{L_{p}\left(Q_{\pi}\right)}}, \\
& E_{p, q, D_{N}, V}:=\sup _{f \in\left(B_{V} \cap L_{p}\left(\mathbb{R}^{m}\right)\right) \backslash\{0\}} \frac{\left\|D_{N}(f)\right\|_{L_{q}\left(\mathbb{R}^{m}\right)}}{\|f\|_{L_{p}\left(\mathbb{R}^{m}\right)}},
\end{aligned}
$$

where $0<p \leq q \leq \infty, Q_{\pi}=\left\{x \in \mathbb{R}^{m}:\left|x_{j}\right| \leq \pi, 1 \leq j \leq m\right\}$, and $D_{N}=\sum_{|\alpha|=N} b_{\alpha} D^{\alpha}$ is a differential operator with constant coefficients. We prove that$$
E_{p, q, D_{N}, V} \leq \liminf _{a \rightarrow \infty} P_{p, q, D_{N}, a, V}, \quad E_{p, \infty, D_{N}, V}=\lim _{a \rightarrow \infty} P_{p, \infty, D_{N}, a, V}
$$

\section{INTROduCtion}

In this paper we discuss relations between the sharp constants in the multivariate BernsteinNikolskii type inequalities for trigonometric polynomials and entire functions of exponential type.

Notation. Let $\mathbb{R}^{m}$ be the Euclidean $m$-dimensional space with elements $x=\left(x_{1}, \ldots, x_{m}\right), y=$ $\left(y_{1}, \ldots, y_{m}\right), u=\left(u_{1}, \ldots, u_{m}\right)$, the inner product $x \cdot y:=\sum_{j=1}^{m} x_{j} y_{j}$, and the norm $|x|:=\sqrt{x \cdot x}$. Next, $\mathbb{C}^{m}:=\mathbb{R}^{m}+i \mathbb{R}^{m}$ is the $m$-dimensional complex space with elements $z=\left(z_{1}, \ldots, z_{m}\right) ; \mathbb{Z}^{m}$ denotes the set of all integral lattice points in $\mathbb{R}^{m}$; and $\mathbb{Z}_{+}^{m}$ is a subset of $\mathbb{Z}^{m}$ of all points with nonnegative coordinates. Given $\sigma \in \mathbb{R}^{m}, \sigma_{j}>0,1 \leq j \leq m$, and $M>0$, let $\Pi_{\sigma}:=\left\{x \in \mathbb{R}^{m}\right.$ : $\left.\left|x_{j}\right| \leq \sigma_{j}, 1 \leq j \leq m\right\}, Q_{M}:=\left\{x \in \mathbb{R}^{m}:\left|x_{j}\right| \leq M, 1 \leq j \leq m\right\}$, and $\mathfrak{B}_{M}:=\left\{x \in \mathbb{R}^{m}:|x| \leq M\right\}$ be the $m$-dimensional parallelepiped, cube, and ball, respectively. In addition, let $d(x, B):=$ $\inf _{y \in B}|x-y|, x \in \mathbb{R}^{m} ; \quad d(A, B):=\inf _{x \in A} d(x, B) ;$ and $\operatorname{diam}(A):=\sup _{x \in A} \sup _{y \in A}|x-y|$, where $A$ and $B$ are subsets of $\mathbb{R}^{m}$.

2010 Mathematics Subject Classification. Primary 41A17, 41A63, Secondary 26D05, 26D10.

Key words and phrases. Sharp constants, multivariate Bernstein-Nikolskii inequality, trigonometric polynomials, entire functions of exponential type, multivariate Levitan's polynomials. 
Let $L_{r}(E)$ be the space of all measurable complex-valued functions $F$ on a measurable set $E \subseteq \mathbb{R}^{m}$ with the finite functional

$$
\|F\|_{L_{r}(E)}:= \begin{cases}\left(\int_{E}|F(x)|^{r} d x\right)^{1 / r}, & 0<r<\infty, \\ {\operatorname{ess} \sup _{x \in E}|F(x)|,} r=\infty .\end{cases}
$$

This functional allows the following "triangle" inequality:

$$
\left\|\sum_{j=1}^{s} F_{j}\right\|_{L_{r}(E)}^{\tilde{r}} \leq \sum_{j=1}^{s}\left\|F_{j}\right\|_{L_{r}(E)}^{\tilde{r}}, \quad F_{j} \in L_{r}(E), \quad 1 \leq j \leq s,
$$

where $s \in \mathbb{N}:=\{1,2, \ldots\}$ and $\tilde{r}:=\min \{1, r\}$ for $r \in(0, \infty]$.

Throughout the paper $V$ is a centrally symmetric (with respect to the origin) closed convex body in $\mathbb{R}^{m}$. Its $m$-dimensional volume is denoted by $|V|_{m}$. The set $V$ generates the following dual norms on $\mathbb{R}^{m}$ and $\mathbb{C}^{m}$ by

$$
\|y\|_{V}^{*}:=\sup _{x \in V}|x \cdot y|, \quad y \in \mathbb{R}^{m} ; \quad\|z\|_{V}^{*}:=\sup _{x \in V}\left|\sum_{j=1}^{m} x_{j} z_{j}\right|, \quad z \in \mathbb{C}^{m} .
$$

For example, if $\sigma \in \mathbb{R}^{m}, \sigma_{j}>0,1 \leq j \leq m$, and $V=\left\{x \in \mathbb{R}^{m}:\left(\sum_{j=1}^{m}\left|x_{j} / \sigma_{j}\right|^{\mu}\right)^{1 / \mu} \leq 1\right\}$, then for $y \in \mathbb{R}^{m},\|y\|_{V}^{*}=\left(\sum_{j=1}^{m}\left|\sigma_{j} y_{j}\right|^{\lambda}\right)^{1 / \lambda}$, where $\mu \in[1, \infty], \lambda \in[1, \infty]$, and $1 / \mu+1 / \lambda=1$. In particular, $\|y\|_{\Pi_{\sigma}}^{*}=\sum_{j=1}^{m} \sigma_{j}\left|y_{j}\right|,\|y\|_{Q_{M}}^{*}=M \sum_{j=1}^{m}\left|y_{j}\right|$, and $\|y\|_{\mathfrak{B}_{M}}^{*}=M|y|$.

Given $a>0$, the set of all trigonometric polynomials $T(x)=\sum_{k \in a V \cap \mathbb{Z}^{m}} c_{k} e^{i k \cdot x}$ with complex coefficients is denoted by $\mathcal{T}_{a V}$.

Definition 1.1. We say that an entire function $f: \mathbb{C}^{m} \rightarrow \mathbb{C}^{1}$ has exponential type $V$ if for any $\varepsilon>0$ there exists a constant $C_{0}(\varepsilon, f)$ such that for all $z \in \mathbb{C}^{m},|f(z)| \leq C_{0}(\varepsilon, f) \exp \left((1+\varepsilon)\|z\|_{V}^{*}\right)$.

The class of all entire function of exponential type $V$ is denoted by $B_{V}$. Throughout the paper, if no confusion may occur, the same notation is applied to $f \in B_{V}$ and its restriction to $\mathbb{R}^{m}$ (e. g., in the form $\left.f \in B_{V} \cap L_{p}\left(\mathbb{R}^{m}\right)\right)$. The class $B_{V}$ was defined by Stein and Weiss [23, Sect. 3.4]. For $V=\Pi_{\sigma}, V=Q_{M}$, and $V=\mathfrak{B}_{M}$, similar classes were defined by Bernstein [3] and Nikolskii [20], [21, Sects. 3.1, 3.2.6], see also [6, Definition 5.1]. Properties of functions from $B_{V}$ have been investigated in numerous publications (see, e. g., [3, 20, 21, 23, 19, 10] and references therein). Some of these properties are presented in Section 2, In particular, if $f \in B_{V} \cap L_{\infty}\left(\mathbb{R}^{m}\right)$, then the norm $\|z\|_{V}^{*}$ in Definition 1.1 can be replaced with $\|\operatorname{Im} z\|_{V}^{*}$ (see Lemma 2.1(b)).

The Fourier transform of a function $\psi \in L_{1}\left(\mathbb{R}^{m}\right)$ or $\psi \in L_{2}\left(\mathbb{R}^{m}\right)$ is denoted by the formula

$$
\widehat{\psi}(y):=\frac{1}{(2 \pi)^{m / 2}} \int_{\mathbb{R}^{m}} \psi(x) e^{-i x \cdot y} d x .
$$


We use the same notation $\widehat{\psi}$ for the Fourier transform of a tempered distribution $\psi$ on $\mathbb{R}^{m}$. By the definition (see, e. g., [23, Sect. 1.3]), $\psi$ is a continuous linear functional $\langle\psi, \varphi\rangle$ on the Schwartz class $S\left(\mathbb{R}^{m}\right)$ of all test functions $\varphi$ on $\mathbb{R}^{m}$, and $\widehat{\psi}$ is defined by the formula $\langle\widehat{\psi}, \varphi\rangle:=\langle\psi, \widehat{\varphi}\rangle, \varphi \in S\left(\mathbb{R}^{m}\right)$. The support of a function or a distribution $\psi$ on $\mathbb{R}^{m}$ is denoted by $\operatorname{supp}(\psi)$.

Throughout the paper $C, C_{0}, C_{1}, \ldots$ denote positive constants independent of essential parameters. Occasionally we indicate dependence on certain parameters.

We also use multi-indices $\alpha=\left(\alpha_{1}, \ldots, \alpha_{m}\right) \in \mathbb{Z}_{+}^{m}$ and $\beta=\left(\beta_{1}, \ldots, \beta_{m}\right) \in \mathbb{Z}_{+}^{m}$ with $|\alpha|:=$ $\sum_{j=1}^{m} \alpha_{j}, \beta \leq \alpha$ (i. e., $\left.\beta_{j} \leq \alpha_{j}, 1 \leq j \leq m\right)$, and

$$
\left(\begin{array}{c}
\alpha \\
\beta
\end{array}\right):=\frac{\prod_{j=1}^{m} \alpha_{j} !}{\prod_{j=1}^{m} \beta_{j} ! \prod_{j=1}^{m}\left(\alpha_{j}-\beta_{j}\right) !}, \quad x^{\alpha}:=x_{1}^{\alpha_{1}} \cdots x_{m}^{\alpha_{m}}, \quad D^{\alpha}:=\frac{\partial^{\alpha_{1}}}{\partial x_{1}^{\alpha_{1}}} \cdots \frac{\partial^{\alpha_{m}}}{\partial x_{m}^{\alpha_{m}}} .
$$

In addition, we use the ceiling and floor functions $\lceil a\rceil$ and $\lfloor a\rfloor$.

Multivariate Bernstein-Nikolskii Type Inequalities. Let $D_{N}:=\sum_{|\alpha|=N} b_{\alpha} D^{\alpha}$ be a linear differential operator with constant coefficients $b_{\alpha} \in \mathbb{C}^{1},|\alpha|=N$, and let $\Delta_{N}(y):=\sum_{|\alpha|=N} b_{\alpha} y^{\alpha}, y \in$ $\mathbb{R}^{m}$, be the total symbol of $D_{N}, N \in \mathbb{N}$. We assume that $D_{0}$ is the identity operator and $\Delta_{0}:=1$.

Next, we define sharp constants in multivariate Bernstein-Nikolskii type inequalities for trigonometric polynomials and entire functions of exponential type. Let

$$
\begin{aligned}
& P_{p, q, D_{N}, a, V}:=a^{-N-m / p+m / q} \sup _{T \in \mathcal{T}_{a V} \backslash\{0\}} \frac{\left\|D_{N}(T)\right\|_{L_{q}\left(Q_{\pi}\right)}}{\|T\|_{L_{p}\left(Q_{\pi}\right)}}, \\
& E_{p, q, D_{N}, V}:=\sup _{f \in\left(B_{V} \cap L_{p}\left(\mathbb{R}^{m}\right)\right) \backslash\{0\}} \frac{\left\|D_{N}(f)\right\|_{L_{q}\left(\mathbb{R}^{m}\right)}}{\|f\|_{L_{p}\left(\mathbb{R}^{m}\right)}} .
\end{aligned}
$$

Here, $a>0, N \in \mathbb{Z}_{+}^{1}, V \subset \mathbb{R}^{m}$, and $0<p \leq q \leq \infty$. Note that

$$
E_{p, q, D_{N}, \gamma V}=\gamma^{N+m / p-m / q} E_{p, q, D_{N}, V}, \quad \gamma>0 .
$$

Indeed, by Definition 1.1. $g \in B_{\gamma V}$ if and only if $f(x)=g(x / \gamma) \in B_{V}$, and (1.4) follows from the relations

$$
\begin{aligned}
E_{p, q, D_{N}, \gamma V} & =\gamma^{N+m / p-m / q} \sup _{g \in\left(B_{\gamma V} \cap L_{p}\left(\mathbb{R}^{m}\right)\right) \backslash\{0\}} \frac{\left\|D_{N}\left(g\left(\gamma^{-1} \cdot\right)\right)\right\|_{L_{q}\left(\mathbb{R}^{m}\right)}}{\left\|g\left(\gamma^{-1} \cdot\right)\right\|_{L_{p}\left(\mathbb{R}^{m}\right)}} \\
& =\gamma^{N+m / p-m / q} \sup _{f \in\left(B_{V} \cap L_{p}\left(\mathbb{R}^{m}\right)\right) \backslash\{0\}} \frac{\left\|D_{N}(f)\right\|_{L_{q}\left(\mathbb{R}^{m}\right)}}{\|f\|_{L_{p}\left(\mathbb{R}^{m}\right)}}
\end{aligned}
$$

A detailed survey of univariate Bernstein-Nikolskii inequalities for $V=[-\sigma, \sigma], D_{N}=d^{N} / d x^{N}, a=$ $n / \sigma, \sigma>0, n \in \mathbb{N}$, and $0<p \leq q \leq \infty$ was presented in [11], so we assume that $m>1$ in all discussions below. 
The Bernstein-Nikolskii sharp constants $P_{p, q, D_{N}, a, V}$ and $E_{p, q, D_{N}, V}$ for $0<p<q \leq \infty$ can be easily found only for $p=2$ and $q=\infty$. Namely,

$$
\begin{aligned}
& P_{2, \infty, D_{N}, a, V}=(2 \pi)^{-m / 2} a^{-(N+m / 2)}\left(\sum_{k \in a V \cap \mathbb{Z}^{m}}\left|\Delta_{N}(i k)\right|^{2}\right)^{1 / 2}, \\
& E_{2, \infty, D_{N}, V}=(2 \pi)^{-m / 2}\left(\int_{V}\left|\Delta_{N}(i x)\right|^{2} d x\right)^{1 / 2} .
\end{aligned}
$$

For $N=0$ these equalities were obtained by Nessel and Wilmes [19, pp. 10, 11]. The Bernstein sharp constants for $0<p=q \leq \infty$ can be found in some special cases as well. In particular,

$$
\begin{aligned}
& P_{2,2, D_{N}, a, V}=a^{-N} \max _{k \in a V \cap \mathbb{Z}^{m}}\left|\Delta_{N}(i k)\right|, \quad E_{2,2, D_{N}, V}=\max _{x \in V}\left|\Delta_{N}(i x)\right|, \\
& P_{q, q, D^{\alpha}, a, \Pi_{\sigma}}=\sigma^{\alpha}(1+o(1)), \quad E_{q, q, D^{\alpha}, \Pi_{\sigma}}=\sigma^{\alpha}, \quad|\alpha|=N, \quad q \in(0, \infty], \\
& P_{q, q, D^{\alpha}, a, Q_{M}}=M^{|\alpha|}(1+o(1)), \quad E_{q, q, D^{\alpha}, Q_{M}}=M^{|\alpha|}, \quad|\alpha|=N, \quad q \in(0, \infty],
\end{aligned}
$$

as $a \rightarrow \infty$. Note that the following limit relations hold true:

$$
\begin{aligned}
& E_{2, \infty, D_{N}, V}=\lim _{a \rightarrow \infty} P_{2, \infty, D_{N}, a, V}, \\
& E_{2,2, D_{N}, V}=\lim _{a \rightarrow \infty} P_{2,2, D_{N}, a, V}, \\
& E_{q, q, D^{\alpha}, \Pi_{\sigma}}=\lim _{a \rightarrow \infty} P_{q, q, D^{\alpha}, a, \Pi_{\sigma}}, \\
& E_{q, q, D^{\alpha}, Q_{M}}=\lim _{a \rightarrow \infty} P_{q, q, D^{\alpha}, a, Q_{M}} .
\end{aligned}
$$

Simple proofs of relations (1.5)-(1.11) are presented in Lemmas 2.6(a), 2.7, and 2.8, while (1.12) and (1.13) follow immediately from (1.8) and (1.9), respectively. Certain sharp Bernstein-type inequalities for functions from $B_{V}$ in case of $p=q=\infty$ are known for special $V$ and $D_{N}$. In particular, Kamzolov [15, Corollary 2] proved the relation

$$
E_{\infty, \infty, \Delta, \mathfrak{B}_{M}}=m M^{2}
$$

where $\Delta$ is the Laplace operator. The author [7, Theorem 5], [8, Theorem 2] extended (1.14) to elliptic differential operators $D_{2}$ with constant coefficients and $m$-dimensional ellipsoids $V$.

So there are just a few examples of finding exact or asymptotically exact values of $P_{p, q, D_{N}, a, V}$ and $E_{p, q, D_{N}, V}$ for $0<p \leq q \leq \infty$. However, efficient estimates of these constants are possible.

The following multivariate Nikolskii-type inequalities for $0<p \leq q \leq \infty$ and functions from $\mathcal{T}_{a V}$ and $B_{V}$ were proved by Nessel and Wilmes [19, Theorems 2, 5]:

$$
\begin{aligned}
& P_{p, q, D_{0}, a, V} \leq\left[(\lceil p / 2\rceil /(2 \pi))^{m}|V|_{m}\right]^{1 / p-1 / q}+o(1), \quad a \rightarrow \infty, \\
& E_{p, q, D_{0}, V} \leq\left[(\lceil p / 2\rceil /(2 \pi))^{m}|V|_{m}\right]^{1 / p-1 / q} .
\end{aligned}
$$


For $V=\Pi_{\sigma}$ and $1 \leq p \leq q \leq \infty$ similar inequalities were established by Nikolskii [20], [21, Sects. $3.3 .5,3.4 .3]$.

Combining estimates (1.15) and (1.16) with (1.9), we arrive at the crude Bernstein-Nikolskii type inequalities $\left(a>0, N \in \mathbb{Z}_{+}^{1}, V \subset \mathbb{R}^{m}, 0<p \leq q \leq \infty\right)$

$$
\begin{aligned}
& P_{p, q, D_{N}, a, V} \leq C_{1}(\operatorname{diam}(V))^{N+m / p-m / q}+o(1), \quad a \rightarrow \infty \\
& E_{p, q, D_{N}, V} \leq C_{2}(\operatorname{diam}(V))^{N+m / p-m / q}
\end{aligned}
$$

where $C_{1}$ is independent of $a$ and $V$, and $C_{2}$ is independent of $V$ (see Lemma 2.6(b) for a short proof).

Main Results. Our major results discuss relations between $P_{p, q, D_{N}, a, V}$ and $E_{p, q, D_{N}, V}$. In particular, we extend relation (1.10) to any $p \in(0, \infty)$.

Theorem 1.2. If $a>0, N \in \mathbb{Z}_{+}^{1}, V \subset \mathbb{R}^{m}$, and $0<p \leq q \leq \infty$, then the following relation holds true:

$$
E_{p, q, D_{N}, V} \leq \liminf _{a \rightarrow \infty} P_{p, q, D_{N}, a, V}
$$

In case of $q=\infty$ a more precise result is valid.

Theorem 1.3. If $a>0, N \in \mathbb{Z}_{+}^{1}, V \subset \mathbb{R}^{m}$, and $p \in(0, \infty]$, then $\lim _{a \rightarrow \infty} P_{p, \infty, D_{N}, a, V}$ exists and

$$
E_{p, \infty, D_{N}, V}=\lim _{a \rightarrow \infty} P_{p, \infty, D_{N}, a, V}
$$

In addition, there exists a nontrivial function $f_{0} \in B_{V} \cap L_{p}\left(\mathbb{R}^{m}\right)$ such that

$$
\left\|D_{N}\left(f_{0}\right)\right\|_{L_{\infty}\left(\mathbb{R}^{m}\right)} /\left\|f_{0}\right\|_{L_{p}\left(\mathbb{R}^{m}\right)}=\lim _{a \rightarrow \infty} P_{p, \infty, D_{N}, a, V}
$$

Remark 1.4. Relations (1.20) and (1.21) show that the function $f_{0} \in B_{V} \cap L_{p}\left(\mathbb{R}^{m}\right)$ from Theorem 1.3 is an extremal function for $E_{p, \infty, D_{N}, V}$.

Remark 1.5. In view of (1.20), (1.11), and (1.12) we believe that the following conjecture is valid.

Conjecture 1.6. The limit $\lim _{a \rightarrow \infty} P_{p, q, D_{N}, a, V}$ exists and $E_{p, q, D_{N}, V}=\lim _{a \rightarrow \infty} P_{p, q, D_{N}, a, V}$ for $0<$ $p \leq q<\infty$.

Remark 1.7. In definitions (1.2) and (1.3) of the sharp constants we discuss only complex-valued functions $T$ and $f$. We can define similarly the "real" sharp constants if the suprema in (1.2) and (1.3) are taken over all real-valued functions on $\mathbb{R}^{m}$ from $\mathcal{T}_{a V} \backslash\{0\}$ and $\left(B_{V} \cap L_{p}\left(\mathbb{R}^{m}\right)\right) \backslash\{0\}$. 
We do not know as to whether the "complex" and "real" sharp constants coincide but for $q=\infty$ this is true. For $m=1$ this fact was proved in [11, Theorem 1.1] and the case of $m>1$ can be proved similarly.

"Real" analogues of Theorems 1.2 and 1.3 are valid as well. The proof of the "real" version of (1.19) is similar to that of Theorem 1.2 if we use Property 3.2 from Section 3 , The "real" version of (1.20) follows immediately from the fact mentioned above. The "real" version of Conjecture 1.6 is believed to be true as well.

Remark 1.8. In the univariate case of $V=[-1,1], D_{N}=d^{N} / d x^{N}$, and $a \in \mathbb{N}$, Theorems 1.2 and 1.3 for the "real" and "complex" sharp constants were proved by the author and Tikhonov [11]. In earlier publications [16, 17], Levin and Lubinsky established versions of Theorems 1.2 and 1.3 on the unit circle for $N=0$. More precise asymptotics for $P_{p, \infty, D_{0}, a,[-1,1]}$ were obtained by Gorbachev and Martyanov [12, Theorem 1]. Certain extensions of the Levin-Lubinsky's results to the $m$-dimensional unit sphere in $\mathbb{R}^{m+1}$ were recently proved by Dai, Gorbachev, and Tikhonov [ 5 , Theorem 1.1].

The proofs of Theorems 1.2 and 1.3 are presented in Section 4 . Section 2 contains certain properties of functions from $B_{V}$ and $\mathcal{T}_{a V}$. Multivariate Levitan's polynomials are introduced in Section 3 ,

\section{Properties of Entire Functions and Trigonometric Polynomials}

In this section we discuss certain properties of functions from $B_{V}$ and $\mathcal{T}_{a V}$ that are needed for the proofs of Theorems 1.2 and 1.3 (see Lemmas 2.1, 2.3, 2.4). In addition, we prove here certain multivariate Bernstein-Nikolskii type inequalities presented in Section 1 (see Lemmas 2.5 through 2.8). Certain standard facts are included in the following lemma.

Lemma 2.1. (a) If $V_{1} \subseteq V_{2}$, then $\mathcal{T}_{a V_{1}} \subseteq \mathcal{T}_{a V_{2}}$ and $B_{V_{1}} \subseteq B_{V_{2}}$.

(b) If $f \in B_{V} \cap L_{\infty}\left(\mathbb{R}^{m}\right)$, then for every $x \in \mathbb{R}^{m}$ and $y \in \mathbb{R}^{m}$,

$$
|f(x+i y)| \leq\|f\|_{L_{\infty}\left(\mathbb{R}^{m}\right)} \exp \left(\|y\|_{V}^{*}\right) .
$$

(c) Let $f$ be a tempered distribution on $\mathbb{R}^{m}$.

(i) If $f \in B_{V}$, then $\operatorname{supp}(\hat{f}) \subseteq V$.

(ii) If $\operatorname{supp}(\widehat{f}) \subseteq V$, then $f$ can be extended to $\mathbb{C}^{m}$ as a function from $B_{V}$.

(d) Let $f$ be a tempered distribution on $\mathbb{R}^{m}$. If $f \in B_{V}$, then $D^{\alpha} f \in B_{V}$ for every $\alpha \in \mathbb{Z}_{+}^{m}$. 
Proof. Statement (a) of the lemma follows immediately from the definitions of $\mathcal{T}_{a V}$ and $B_{V}$. Statement (b) is established in [19, Eq. (4.13)] (cf. [23, Lemma 3.4.11]), while the proof of a PaleyWiener-Schwartz type theorem (c) is outlined in [19, p. 13]. It remains to prove statement (d). Let $\varphi$ be a test function from $S\left(\mathbb{R}^{m}\right)$ with $\operatorname{supp}(\varphi) \subseteq \mathbb{R}^{m} \backslash V$. Then for any $\alpha \in \mathbb{Z}_{+}^{m}$, the function $\varphi_{1}(x):=x^{\alpha} \varphi(x)$ belongs to $S\left(\mathbb{R}^{m}\right)$ and $\operatorname{supp}\left(\varphi_{1}\right) \subseteq \mathbb{R}^{m} \backslash V$. Therefore, by part (i) of statement (c),

$$
\left\langle\widehat{D^{\alpha} f}, \varphi\right\rangle=i^{|\alpha|}\left\langle\widehat{f}, \varphi_{1}\right\rangle=0
$$

So $\operatorname{supp}\left(\widehat{D^{\alpha}} f\right) \subseteq V$, and by part (ii) of statement (c), $D^{\alpha} f \in B_{V}$.

Remark 2.2. The condition in statements (c) and (d) of Lemma 2.1 that $f$ is a tempered distribution on $\mathbb{R}^{m}$ is obviously satisfied for $f \in L_{p}\left(\mathbb{R}^{m}\right), p \in(0, \infty]$. In particular, when $f \in L_{2}\left(\mathbb{R}^{m}\right)$, a PaleyWiener type theorem of Lemma 2.1 (c) was proved in [23, Theorem 3.4.9].

The compactness theorem for the set $B_{V} \cap L_{\infty}\left(\mathbb{R}^{m}\right)$ is discussed below.

Lemma 2.3. For any sequence $\left\{f_{n}\right\}_{n=1}^{\infty}, f_{n} \in B_{V} \cap L_{\infty}\left(\mathbb{R}^{m}\right), n \in \mathbb{N}$, with $\sup _{n \in \mathbb{N}}\left\|f_{n}\right\|_{L_{\infty}\left(\mathbb{R}^{m}\right)}=C$, there exist a subsequence $\left\{f_{n_{s}}\right\}_{s=1}^{\infty}$ and a function $f_{0} \in B_{V} \cap L_{\infty}\left(\mathbb{R}^{m}\right)$ such that for every $\alpha \in \mathbb{Z}_{+}^{m}$,

$$
\lim _{s \rightarrow \infty} D^{\alpha} f_{n_{s}}=D^{\alpha} f_{0}
$$

uniformly on any compact set in $\mathbb{C}^{m}$.

Proof. For $V=\Pi_{\sigma}$ this compactness result was proved by Nikolskii [21, Theorem 3.3.6]. Next, for any $V \subset \mathbb{R}^{m}$ there exists $\sigma(V) \in \mathbb{R}^{m}, \sigma(V)_{j}>0,1 \leq j \leq m$, such that $V \subseteq \Pi_{\sigma(V)}$. Note that by Lemma 2.1(a), $f_{n} \in B_{\Pi_{\sigma(V)}} \cap L_{\infty}\left(\mathbb{R}^{m}\right), n \in \mathbb{N}$. Then by the Nikolskii's compactness theorem, there exists a subsequence $\left\{f_{n_{s}}\right\}_{s=1}^{\infty}$ and a function $f_{0} \in B_{\Pi_{\sigma(V)}} \cap L_{\infty}\left(\mathbb{R}^{m}\right)$ such that (2.1) holds true uniformly on any compact set in $\mathbb{C}^{m}$. It remains to show that $f_{0} \in B_{V} \cap L_{\infty}\left(\mathbb{R}^{m}\right)$. Indeed, by Lemma 2.1(b),

$$
\left|f_{n}(x+i y)\right| \leq C \exp \left(\|y\|_{V}^{*}\right), \quad n \in \mathbb{N}, \quad x \in \mathbb{R}^{m}, \quad y \in \mathbb{R}^{m} .
$$

Therefore using first (2.1) for $\alpha=0$ and then (2.2), we obtain for any $x \in \mathbb{R}^{m}$ and $y \in \mathbb{R}^{m}$

$$
\left|f_{0}(x+i y)\right|=\lim _{s \rightarrow \infty}\left|f_{n_{s}}(x+i y)\right| \leq C \exp \left(\|y\|_{V}^{*}\right) \leq C \exp \left(\|x+i y\|_{V}^{*}\right) .
$$

Thus $f_{0} \in B_{V} \cap L_{\infty}\left(\mathbb{R}^{m}\right)$.

Periodization properties of functions from $B_{V}$ are based on the following version of the Poisson summation formula (cf. [23, Sect. 7.2]). 
Lemma 2.4. If $g \in L_{1}\left(\mathbb{R}^{m}\right)$ and the series $\sum_{k \in \mathbb{Z}^{m}} \widehat{g}(k) e^{i k \cdot y}$ is a finite sum, then the series $\sum_{k \in \mathbb{Z}^{m}} g(y+2 k \pi)$ converges in $L_{1}\left(Q_{\pi}\right)$ to a function from $L_{1}\left(Q_{\pi}\right)$ and

$$
\sum_{k \in \mathbb{Z}^{m}} g(y+2 k \pi)=(2 \pi)^{-m / 2} \sum_{k \in \mathbb{Z}^{m}} \widehat{g}(k) e^{i k \cdot y} .
$$

Proof. It was proved in [23, Theorem 7.2.4]) that if $g \in L_{1}\left(\mathbb{R}^{m}\right)$, then $\sum_{k \in \mathbb{Z}^{m}} g(y+2 k \pi)$ converges in $L_{1}\left(Q_{\pi}\right)$ to a function $\tilde{g} \in L_{1}\left(Q_{\pi}\right)$ and its Fourier series expansion coincides with the right-hand side of (2.3) . Since it is a trigonometric polynomial $T$, we conclude that $\tilde{g}=T$.

Lemma 2.5. For any $M>0$ and $q \in(0, \infty]$ there exists a family $\left\{f_{h, q, M}\right\}_{h \in(0, M)}=\left\{f_{h}\right\}_{h \in(0, M)}$ of functions from $B_{[-M, M]}$ such that for any $s \in \mathbb{N}$,

$$
\frac{\left\|f_{h}^{(s)}\right\|_{L_{q}\left(\mathbb{R}^{1}\right)}}{\left\|f_{h}\right\|_{L_{q}\left(\mathbb{R}^{1}\right)}} \geq M^{s}(1-o(1)), \quad h \rightarrow 0^{+} .
$$

Proof. For $q \in[1, \infty]$ and $s=1$ this result was proved by Akhiezer [1, Sect. 84]. We will use the similar construction. Let $d:=\lfloor 1 / q\rfloor+1$ and let $\varphi$ be a continuously $d$-differentiable function on $[0,1]$, satisfying the following boundary conditions: $\varphi^{(l)}(0)=\varphi^{(l)}(1)=0,0 \leq l \leq d$. Then the function

$$
f_{h}(t):=e^{i M t} \int_{0}^{1} e^{-i h t \tau} \varphi(\tau) d \tau=\frac{1}{h} \int_{M-h}^{M} e^{i t \tau} \varphi\left(\frac{M-\tau}{h}\right) d \tau, \quad h \in(0, M),
$$

belongs to $B_{[-M, M]} \cap L_{q}\left(\mathbb{R}^{1}\right)$ because integration by parts in the first integral shows that

$$
\left|f_{h}(t)\right| \leq \min \left\{\int_{0}^{1}|\varphi(\tau)| d \tau, \frac{1}{(h t)^{d}} \int_{0}^{1}\left|\varphi^{(d)}(\tau)\right| d \tau\right\}, \quad t \in \mathbb{R}^{1} .
$$

Next,

$$
f_{h}^{(s)}(t)=i^{s} e^{i M t} \sum_{l=0}^{s}(-1)^{s-l}\left(\begin{array}{l}
s \\
l
\end{array}\right) M^{l} h^{s-l} \psi_{s-l}(h t)
$$

where

$$
\psi_{r}(t):=\int_{0}^{1} e^{-i t \tau} \tau^{r} \varphi(\tau) d \tau, \quad 0 \leq r \leq s .
$$

Note that $\psi_{r} \in B_{[-1,1]} \cap L_{q}\left(\mathbb{R}^{1}\right), 0 \leq r \leq s$, by Bernstein's inequality, since $\left|\psi_{0}(h t)\right|=\left|f_{h}(t)\right|$ and $\left|\psi_{r}(t)\right|=\left|\psi_{0}^{(r)}(t)\right|$. Then we have by (1.1)

$$
\frac{\left\|f_{h}^{(s)}\right\|_{L_{q}\left(\mathbb{R}^{1}\right)}^{\tilde{q}}}{\left\|f_{h}\right\|_{L_{q}\left(\mathbb{R}^{1}\right)}^{\tilde{q}}} \geq M^{s \tilde{q}}-h^{\tilde{q}} \sum_{l=0}^{s-1}\left(\begin{array}{c}
s \\
l
\end{array}\right)^{\tilde{q}} M^{l \tilde{q}} h^{(s-l-1) \tilde{q}} \frac{\left\|\psi_{s-l}\right\|_{L_{q}\left(\mathbb{R}^{1}\right)}^{\tilde{q}}}{\left\|\psi_{0}\right\|_{L_{q}\left(\mathbb{R}^{1}\right)}^{\tilde{q}}}=M^{s \tilde{q}}(1-o(1))^{\tilde{q}}, \quad h \rightarrow 0^{+} .
$$

This establishes the lemma.

Lemma 2.6. (a) Relations (1.8) and (1.9) hold true. (b) Inequalities (1.17) and (1.18) are valid. 
Proof. (a) We first note that in the univariate case

$$
E_{q, q, d^{s}} / d x^{s},[-M, M]=M^{s}, \quad q \in(0, \infty], \quad s \in \mathbb{N} .
$$

Indeed, the proof of the classical inequality $E_{q, q, d^{s} / d x^{s},[-M, M]} \leq M^{s}$ can be found in [4, Theorem 11.3.3] for $q \in[1, \infty]$ and in [22] for $q \in(0,1)$. Then (2.4) follows from Lemma 2.5. Next, it follows from (2.4) that for every $f \in B_{\Pi_{\sigma}} \cap L_{q}\left(\mathbb{R}^{m}\right), q \in(0, \infty], \alpha_{j} \in \mathbb{Z}_{+}^{1}$, and $1 \leq j \leq m$,

$$
\left(\int_{R^{1}}\left|\frac{\partial^{\alpha_{j}} f\left(x_{1}, \ldots, x_{j}, \ldots, x_{m}\right)}{\partial x_{j}^{\alpha_{j}}}\right|^{q} d x_{j}\right)^{1 / q} \leq \sigma_{j}^{\alpha_{j}}\left(\int_{\mathbb{R}^{1}}\left|f\left(x_{1}, \ldots, x_{j}, \ldots, x_{m}\right)\right|^{q} d x_{j}\right)^{1 / q} .
$$

Therefore,

$$
\left\|\frac{\partial^{\alpha_{j}} f}{\partial x_{j}^{\alpha_{j}}}\right\|_{L_{q}\left(\mathbb{R}^{m}\right)} \leq \sigma_{j}^{\alpha_{j}}\|f\|_{L_{q}\left(\mathbb{R}^{m}\right)}, \quad 1 \leq j \leq m .
$$

Using this inequality $m$ times, we arrive at the inequality

$$
E_{q, q, D^{\alpha}, \Pi_{\sigma}} \leq \sigma^{\alpha}
$$

This inequality is well-known for $q \in[1, \infty]$ (see, e. g., [21, Eq. (3.2.2.8)]). Finally, let $f_{h, q, M}$ be functions from Lemma 2.5. Then the functions $F_{h}:=\prod_{j=1}^{m} f_{h, q, \sigma_{j}}\left(x_{j}\right), h \in\left(0, \min _{1 \leq j \leq m} \sigma_{j}\right)$, satisfy the inequality

$$
\left\|D^{\alpha} F_{h}\right\|_{L_{q}\left(\mathbb{R}^{m}\right)} \geq \sigma^{\alpha}(1-o(1))\left\|F_{h}\right\|_{L_{q}\left(\mathbb{R}^{m}\right)}, \quad h \rightarrow 0^{+}, \quad q \in(0, \infty] .
$$

Thus the second equality in (1.8) follows from (2.7) and (2.8).

It is well known that a periodic analog of (2.4) for $a M \in \mathbb{N}$ is

$$
P_{q, q, d^{s}} / d x^{s}, a,[-M, M]=M^{s}, \quad q \in(0, \infty], \quad s \in \mathbb{N},
$$

see, e. g., [24, Sect. 4.8.62] for $q \in[1, \infty]$ and see [2] for $q \in(0,1)$. An extremal polynomial in (2.9) is $\cos (a M \tau)$. Then similarly to (2.4) - (2.7), we obtain from (2.9)

$$
P_{q, q, D^{\alpha}, a, \Pi_{\sigma}} \leq a^{-|\alpha|} \prod_{j=1}^{m}\left\lceil a \sigma_{j}\right\rceil^{\alpha_{j}}=\sigma^{\alpha}(1+o(1)), \quad a \rightarrow \infty .
$$

Finally, the polynomial $T(x):=\prod_{j=1}^{m} \cos \left(\left\lfloor a \sigma_{j}\right\rfloor x_{j}\right)$ satisfies the inequality

$$
P_{q, q, D^{\alpha}, a, \Pi_{\sigma}} \geq a^{-|\alpha|} \frac{\left\|D^{\alpha} T\right\|_{L_{q}\left(Q_{\pi}\right)}}{\|T\|_{L_{q}\left(Q_{\pi}\right)}}=a^{-|\alpha|} \prod_{j=1}^{m}\left(\left\lfloor a \sigma_{j}\right\rfloor\right)^{\alpha_{j}}=\sigma^{\alpha}(1+o(1)), \quad a \rightarrow \infty .
$$

Thus the first equality in (1.8) follows from (2.10) and (2.11). Relations (1.9) follow immediately from (1.8). This completes the proof of statement (a).

(b) Setting $M=\operatorname{diam}(V) / 2$, we see that $V \subseteq \mathfrak{B}_{M} \subseteq Q_{M}$. In particular, $|V|_{m} \leq\left|\mathfrak{B}_{1}\right|_{m}(\operatorname{diam}(V) / 2)^{m}$. 
Then using (1.1), Lemma 2.1(a), and the first relation of (1.9) (see Lemma 2.6(a) for the proof), we obtain

$$
\begin{aligned}
P_{q, q, D_{N}, a, V} & \leq P_{q, q, D_{N}, a, Q_{M}} \leq\left(\sum_{|\alpha|=N}\left|b_{\alpha}\right|^{\tilde{q}} P_{q, q, D^{\alpha}, a, Q_{M}}^{\tilde{q}}\right)^{1 / \tilde{q}} \\
& =\left(\frac{\operatorname{diam}(V)}{2}\right)^{N}\left(\sum_{|\alpha|=N}\left|b_{\alpha}\right|^{\tilde{q}}\right)^{1 / \tilde{q}}+o(1)
\end{aligned}
$$

as $a \rightarrow \infty$. Taking account of the relations

$$
\begin{aligned}
P_{p, q, D_{N}, a, V} & =\sup _{T \in \mathcal{T}_{a V} \backslash\{0\}}\left(a^{-N} \frac{\left\|D_{N}(T)\right\|_{L_{q}\left(Q_{\pi}\right)}}{\|T\|_{L_{q}\left(Q_{\pi}\right)}}\right)\left(a^{-m / p+m / q} \frac{\|T\|_{L_{q}\left(Q_{\pi}\right)}}{\|T\|_{L_{p}\left(Q_{\pi}\right)}}\right) \\
& \leq P_{q, q, D_{N}, a, V} P_{p, q, D_{0}, a, V},
\end{aligned}
$$

we arrive at (1.17) from (2.12) and (1.15). Inequality (1.18) can be proved similarly.

Lemma 2.7. Relations (1.5), (1.6), and (1.7) hold true.

Proof. The proofs of (1.6) and the second relation in (1.7) are based on the Paley-Wiener type theorem (see Lemma 2.1(c) and Remark 2.2). Let $f \in B_{V} \cap L_{2}\left(\mathbb{R}^{m}\right)$. Then

$$
f(u)=\frac{1}{(2 \pi)^{m / 2}} \int_{V} g(x) e^{i u \cdot x} d x, \quad D_{N}(f)(u)=\frac{1}{(2 \pi)^{m / 2}} \int_{V} g(x) \Delta_{N}(i x) e^{i u \cdot x} d x,
$$

where $\|f\|_{L_{2}\left(\mathbb{R}^{m}\right)}=\|g\|_{L_{2}(V)}$ and $\left\|D_{N}(f)\right\|_{L_{2}\left(\mathbb{R}^{m}\right)}=\left\|g \Delta_{N}(i \cdot)\right\|_{L_{2}(V)}$. Therefore,

$$
E_{2,2, D_{N}, V}=\sup _{g \in L_{2}(V) \backslash\{0\}} \frac{\left\|g \Delta_{N}(i \cdot)\right\|_{L_{2}(V)}}{\|\left. g\right|_{L_{2}(V)}}=\left\|\Delta_{N}(i \cdot)\right\|_{L_{\infty}(V)} .
$$

Next, it is easy to see that

$$
\begin{aligned}
E_{2, \infty, D_{N}, V} & =\sup _{f \in\left(B_{V} \cap L_{2}(V)\right) \backslash\{0\}} \frac{\left|D_{N}(f)(0)\right|}{\|f\|_{L_{2}\left(\mathbb{R}^{m}\right)}} \\
& =\frac{1}{(2 \pi)^{m / 2}} \sup _{g \in L_{2}(V) \backslash\{0\}} \frac{\left|\int_{V} g(x) \Delta_{N}(i x) d x\right|}{\|g\|_{L_{2}(V)}} \\
& =\frac{1}{(2 \pi)^{m / 2}}\left\|\Delta_{N}(i \cdot)\right\|_{L_{2}(V)} .
\end{aligned}
$$

The corresponding results for trigonometric polynomials can be proved similarly by using Parseval's identity.

Lemma 2.8. Limit relations (1.10) and (1.11) hold true. 
Proof. Let $\left\|\Delta_{N}(i \cdot)\right\|_{L_{\infty}(V)}=\left|\Delta_{N}\left(i x_{0}\right)\right|$ for a point $x_{0} \in V$. Given $a>0$, we define the set $A(a):=\left\{x \in V: x=k / a, k \in \mathbb{Z}^{m}\right\}$. Then $d\left(x_{0}, A(a)\right) \leq \sqrt{m} / a$. Therefore, for any $\varepsilon>0$ there exist $a_{0}>0$ and $x_{0, a}=k_{a} / a \in A(a)$ such that for all $a>a_{0}, \mid \Delta_{N}\left(i x_{0}\right)-\Delta_{N}\left(i x_{0, a} \mid<\varepsilon\right.$. Hence

$$
\left\|\Delta_{N}(i \cdot)\right\|_{L_{\infty}(V)}<\left|\Delta_{N}\left(i x_{0, a}\right)\right|+\varepsilon \leq a^{-N} \max _{k \in a V \cap \mathbb{Z}^{m}}\left|\Delta_{N}(i k)\right|+\varepsilon \leq\left\|\Delta_{N}(i \cdot)\right\|_{L_{\infty}(V)}+\varepsilon .
$$

Then (1.11) follows from (1.7) and (2.13).

Further, we assume that $a>1$. Let $Q_{M / a}$ be a cube with $M \in \mathbb{N}$, satisfying the conditions $V \subset Q_{M / a}$ and $d\left(\partial Q_{M / a}, V\right)>\sqrt{m}$. It is easy to see that if $Q$ is a cube in $\mathbb{R}^{m}$ with an edge length of $1 / a$ such that $Q \cap V \neq \emptyset$, then $Q \subseteq Q_{M / a}$. Next denoting by $\chi_{V}$ the characteristic function of the set $V$, we see that the sum

$$
\frac{1}{a^{2 N+m}} \sum_{k \in a V \cap \mathbb{Z}^{m}}\left|\Delta_{N}(i k)\right|^{2}=\frac{1}{a^{m}} \sum_{k \in a V \cap \mathbb{Z}^{m}}\left|\Delta_{N}\left(\frac{i k}{a}\right)\right|^{2}=\frac{1}{a^{m}} \sum_{k / a \in Q_{M / a} \cap \mathbb{Z}^{m}}\left|\Delta_{N}\left(\frac{i k}{a}\right)\right|^{2} \chi_{V}\left(\frac{k}{a}\right),
$$

is the Riemann sum of the function $\left|\Delta_{N}(i \cdot)\right|^{2} \chi_{V}(\cdot): Q_{M / a} \rightarrow \mathbb{R}^{1}$, corresponding to the partition

$$
\left\{\left\{x \in \mathbb{R}^{m}: \frac{k_{j}-1}{a} \leq x_{j} \leq \frac{k_{j}}{a}, 1 \leq j \leq m\right\}, k_{j} \in \mathbb{Z}^{1},-M+1 \leq k_{j} \leq M, 1 \leq j \leq m\right\}
$$

of $Q_{M / a}$. Therefore, this sum converges to $\int_{V}\left|\Delta_{N}(i x)\right|^{2} d x$ as $a \rightarrow \infty$. This establishes (1.10).

\section{Multivariate Levitan's Polynomials}

To prove Theorem 1.2, we need a multivariate version of Levitan's trigonometric polynomials introduced in the univariate case by Levitan [18] and Hörmander [13, 14]. Let us define these polynomials and study their properties.

Let us set

$$
h(y):=\prod_{j=1}^{m} \frac{\sin y_{j}}{y_{j}}, \quad y \in \mathbb{R}^{m} .
$$

Then $h^{2}(\cdot / 2) \in B_{Q_{1}} \cap L_{1}\left(\mathbb{R}^{m}\right)$ and

$$
\sum_{k \in \mathbb{Z}^{m}} h^{2}(x / 2+k \pi)=\sum_{k_{1}=-\infty}^{\infty}\left(\frac{\sin \left(x_{1} / 2\right)}{x_{1} / 2+k_{1} \pi}\right)^{2} \cdots \sum_{k_{m}=-\infty}^{\infty}\left(\frac{\sin \left(x_{m} / 2\right)}{x_{m} / 2+k_{m} \pi}\right)^{2}=1, \quad x \in \mathbb{R}^{m} .
$$

Note that for $m=1$ relations (3.2) follows from a well-known expansion of $\sin ^{-2}(\tau / 2), \tau \in \mathbb{R}^{1}$.

Let $f \in B_{V} \cap L_{\infty}\left(\mathbb{R}^{m}\right)$. Then for a fixed number $a>0$ the function $g_{a}(u):=f(u) h^{2}(u /(2 a))$ is an entire function and by Lemma 2.1(b),

$$
\left|g_{a}(x+i y)\right| \leq\|f\|_{L_{\infty}\left(\mathbb{R}^{m}\right)} \exp \left(\|y\|_{V}^{*}+a^{-1} \sum_{j=1}^{m}\left|y_{j}\right|\right) \leq\|f\|_{L_{\infty}\left(\mathbb{R}^{m}\right)} \exp \left((1+c / a)\|y\|_{V}^{*}\right),
$$


where

$$
c:=\sup _{y \in \mathbb{R}^{m} \backslash\{0\}} \frac{\sum_{j=1}^{m}\left|y_{j}\right|}{\|y\|_{V}^{*}} .
$$

Therefore, $g_{a} \in B_{(1+c / a) V} \cap L_{1}\left(\mathbb{R}^{m}\right)$ and by Nikolskii-type inequality (1.16),$g_{a} \in L_{2}\left(\mathbb{R}^{m}\right) \cap B_{(1+c / a) V}$. Then by Lemma 2.1(c), supp $\widehat{g_{a}} \subseteq(1+c / a) V$. Hence the Fourier series

$$
(2 \pi)^{-m / 2} \sum_{k \in \mathbb{Z}^{m}} a^{-m} \widehat{g_{a}}(k / a) e^{i k \cdot x / a}
$$

coincides with a trigonometric polynomial

$$
S_{a}(x)=S_{a}(f, x):=(2 \pi)^{-m / 2} \sum_{k \in(a+c) V \cap \mathbb{Z}^{m}} a^{-m} \widehat{g_{a}}(k / a) e^{i k \cdot x / a}
$$

of period $2 \pi a$ in each variable with its spectrum in $(a+c) V$. This polynomial $S_{a}=S_{a}(f, \cdot)$ we call the multivariate Levitan's polynomial for $f$.

For $m=1, V=[-\sigma, \sigma]$, and $a=n / \sigma, n \in \mathbb{N}$, a trigonometric polynomial $S_{a}$ of degree $n$ was introduced in [18, 13, 14] (see also [1, Sect. 85]). In the case when $V$ is a parallelohedron, multivariate Levitan's polynomials were introduced in [9].

Let us discuss properties of $S_{a}$.

Property 3.1. If $f \in B_{V} \cap L_{\infty}\left(\mathbb{R}^{m}\right)$, then the following representation holds true:

$$
S_{a}(f, x)=\sum_{k \in \mathbb{Z}^{m}} f(x+2 k \pi a) h^{2}(x /(2 a)+k \pi), \quad x \in \mathbb{R}^{m},
$$

where $h$ is defined in (3.1).

Proof. Setting $g(y):=f(a y) h^{2}(y / 2)=g_{a}(a y)$, we see that $g \in L_{1}\left(\mathbb{R}^{m}\right)$ and the series

$$
\sum_{k \in \mathbb{Z}^{m}} \widehat{g}(k) e^{i k \cdot y}=\sum_{k \in \mathbb{Z}^{m}} a^{-m} \widehat{g_{a}}(k / a) e^{i k \cdot y}=(2 \pi)^{m / 2} S_{a}(a y)
$$

is a finite sum. Then using Lemma 2.4, we conclude that (2.3) and (3.6) imply (3.5) for $y=x / a$.

Property 3.2. If $f$ is a real-valued function from $B_{V} \cap L_{\infty}\left(\mathbb{R}^{m}\right)$, then $S_{a}(f, \cdot)$ is a real-valued polynomial.

This property follows immediately from representation (3.5).

Property 3.3. If $f \in B_{V} \cap L_{p}\left(\mathbb{R}^{m}\right), p \in(0, \infty]$, then

$$
\left\|S_{a}\right\|_{L_{p}\left(Q_{a \pi}\right)} \leq\|f\|_{L_{p}\left(\mathbb{R}^{m}\right)}
$$


Proof. We first note that $f \in B_{V} \cap L_{\infty}\left(\mathbb{R}^{m}\right)$ by Nikolskii-type inequality (1.16), so by Property 3.1, $S_{a}$ is represented by (3.5). If $p \in(0, \infty)$, then

$$
\left|S_{a}(x)\right|^{p} \leq \sum_{k=-\infty}^{\infty}|f(x+2 k \pi a)|^{p} .
$$

Indeed, for $p=1$ (3.8) follows immediately from (3.5) since $\|h\|_{L_{\infty}\left(\mathbb{R}^{m}\right)} \leq 1$. If $p \in(1, \infty)$, then by Hölder's inequality, we obtain from relations (3.5) and (3.2) that

$$
\begin{aligned}
\left|S_{a}(x)\right| & \leq\left(\sum_{k \in \mathbb{Z}^{m}}|f(x+2 k \pi a)|^{p}\right)^{1 / p}\left(\sum_{k \in \mathbb{Z}^{m}}|h(x /(2 a)+k \pi)|^{2 p /(p-1)}\right)^{(p-1) / p} \\
& \leq\left(\sum_{k \in \mathbb{Z}^{m}}|f(x+2 k \pi a)|^{p}\right)^{1 / p} .
\end{aligned}
$$

If $p \in(0,1)$, then we have from (3.5)

$$
\left|S_{a}(x)\right|^{p} \leq \sum_{k \in \mathbb{Z}^{m}}|f(x+2 k \pi a)|^{p}|h(x /(2 a)+k \pi)|^{2 p} \leq \sum_{k \in \mathbb{Z}^{m}}|f(x+2 k \pi a)|^{p} .
$$

Thus (3.8) holds true for $p \in(0, \infty)$. Next, integrating (3.8) over $Q_{a \pi}$ and using Fatou's Lemma, we arrive at (3.7) for $p \in(0, \infty)$. Finally, for $p=\infty$ (3.7) follows immediately from (3.5) and (3.2) .

Next, we discuss three approximation properties of multivariate Levitan's polynomials.

Property 3.4. If $f \in B_{V} \cap L_{\infty}\left(\mathbb{R}^{m}\right)$, then for $x \in \mathbb{R}^{m}$,

$$
\left|f(x)-S_{a}(x)\right| \leq C_{3}(|x| / a)^{2}\|f\|_{L_{\infty}\left(\mathbb{R}^{m}\right)},
$$

where $C_{3} \leq 1 / 6$ is an absolute constant.

Proof. To prove (3.9), we need to evaluate certain integrals. First, we note that by Fubini's theorem,

$$
\int_{Q_{1 / 2}}(x \cdot u / a)^{2 l+1} d u=0, \quad a>0, \quad l \in \mathbb{Z}_{+}^{1},
$$

because each monomial in the expansion of $(x \cdot u / a)^{2 l+1}$ contains at least one factor of the form $u_{j}^{\alpha_{j}}$, where $\alpha_{j}>0$ is an odd number, $1 \leq j \leq m$. Then

$$
\int_{Q_{1 / 2}} \sin (x \cdot u / a) d u=\sum_{l=0}^{\infty} \frac{(-1)^{l}}{(2 l+1) !} \int_{Q_{1 / 2}}(x \cdot u / a)^{2 l+1} d u=0 .
$$


Next, it follows from (3.2), (3.5), and (3.10) that

$$
\begin{aligned}
& \left|f(x)-S_{a}(x)\right| \leq|f(x)|\left(1-h^{2}(x /(2 a))\right)+\sum_{k \neq 0}|f(x+2 k \pi a)| h^{2}(x /(2 a)+k \pi) \\
& \leq 2\left(1-\prod_{j=1}^{m}\left(\frac{\sin \left(\frac{x_{j}}{2 a}\right)}{x_{j} /(2 a)}\right)^{2}\right)\|f\|_{L_{\infty}\left(\mathbb{R}^{m}\right)} \leq 4\left(1-\prod_{j=1}^{m} \frac{\sin \left(\frac{x_{j}}{2 a}\right)}{x_{j} /(2 a)}\right)\|f\|_{L_{\infty}\left(\mathbb{R}^{m}\right)} \\
& =4 \int_{Q_{1 / 2}}(1-\exp (i(x \cdot u / a))) d u\|f\|_{L_{\infty}\left(\mathbb{R}^{m}\right)}=4 \int_{Q_{1 / 2}}(1-\cos (x \cdot u / a)) d u\|f\|_{L_{\infty}\left(\mathbb{R}^{m}\right)} \\
& \leq \frac{2}{a^{2}} \int_{Q_{1 / 2}}(x \cdot u)^{2} d u\|f\|_{L_{\infty}\left(\mathbb{R}^{m}\right)}=\frac{|x|^{2}}{6 a^{2}}\|f\|_{L_{\infty}\left(\mathbb{R}^{m}\right)} .
\end{aligned}
$$

Thus (3.9) is established.

Property 3.5. Let $f \in B_{V} \cap L_{\infty}\left(\mathbb{R}^{m}\right)$ and let $D_{N}:=\sum_{|\alpha|=N} b_{\alpha} D^{\alpha}$ be a linear differential operator with constant coefficients $b_{\alpha} \in \mathbb{C}^{1},|\alpha|=N$. Then for $x \in \mathbb{R}^{m}$ and $a \geq 1$,

$$
\left|D_{N}(f)(x)-D_{N}\left(S_{a}\right)(x)\right| \leq C_{4}(\operatorname{diam}(V))^{N}\left(\frac{|x|^{2}}{a^{2}}+\frac{1}{a}\right)\|f\|_{L_{\infty}\left(\mathbb{R}^{m}\right)}
$$

where $C_{4}$ is independent of $x, a, f$, and $V$.

Proof. For $N=0$ Property [3.5 follows from Property [3.4, so we assume that $N \geq 1$. We also note that the uniform convergence of all series below follows easily from identity (3.2), representation (3.5), and Bernstein-type inequality (1.18) for $q=p$. Next, recalling notation $g_{a}(u)=$ $f(u) h^{2}(u /(2 a))$, we see from (3.5) that

$$
\begin{aligned}
& \left|D_{N}(f)(x)-D_{N}\left(S_{a}\right)(x)\right| \\
& \leq\left|D_{N}(f)(x)-\sum_{k \in \mathbb{Z}^{m}} D_{N}(f)(x+2 k \pi a) h^{2}(x /(2 a)+k \pi)\right| \\
& +\left|\sum_{k \in \mathbb{Z}^{m}} D_{N}\left(g_{a}\right)(x+2 k \pi a)-D_{N}(f)(x+2 k \pi a) h^{2}(x /(2 a)+k \pi)\right| \\
& =I_{1}(x)+I_{2}(x) .
\end{aligned}
$$

Since $D_{N}(f) \in B_{V} \cap L_{\infty}\left(\mathbb{R}^{m}\right)$ by Lemma 2.1(d), Remark 2.2, and Bernstein-type inequality (1.18) for $q=p=\infty$, we have from Property 3.4

$$
I_{1}(x)=\left|D_{N}(f)(x)-S_{a}\left(D_{N}(f), x\right)\right| \leq C_{5}(\operatorname{diam}(V))^{N}(|x| / a)^{2}\|f\|_{L_{\infty}\left(\mathbb{R}^{m}\right)}
$$


where $C_{5}$ is independent of $x, a, f$, and $V$. Further, using Bernstein-type inequality (1.18) for $q=p$ again, we obtain by the multivariate Leibniz formula

$$
\begin{aligned}
& I_{2}(x) \\
& \leq \sum_{k \in \mathbb{Z}^{m}} \sum_{|\alpha|=N}\left|b_{\alpha}\right| \sum_{\beta \leq \alpha,|\beta| \geq 1}\left(\begin{array}{c}
\alpha \\
\beta
\end{array}\right)\left|D^{\alpha-\beta}(f)(x+2 k \pi a)\right|\left|D^{\beta}\left(\gamma_{a}\right)(x+2 k \pi a)\right| \\
& \leq C_{6}(1+\operatorname{diam}(V))^{N}\|f\|_{L_{\infty}\left(\mathbb{R}^{m}\right)} \sum_{|\alpha|=N}\left|b_{\alpha}\right| \sum_{\beta \leq \alpha,|\beta| \geq 1} \sum_{k \in \mathbb{Z}^{m}}\left|D^{\beta}\left(\gamma_{a}\right)(x+2 k \pi a)\right| \\
& =C_{6}(1+\operatorname{diam}(V))^{N}\|f\|_{L_{\infty}\left(\mathbb{R}^{m}\right)}(2 a)^{-|\beta|} \sum_{k \in \mathbb{Z}^{m}}\left|D^{\beta}\left(\gamma_{1 / 2}\right)(x /(2 a)+k \pi)\right|,
\end{aligned}
$$

where $\gamma_{a}(u):=h^{2}(u /(2 a))$ and $C_{6}$ is independent of $x, a, f$, and $V$. It remains to estimate the series

$$
\sum_{k \in \mathbb{Z}^{m}}\left|D^{\beta}\left(\gamma_{1 / 2}\right)(y+2 k \pi)\right|=\prod_{j=1}^{m} H_{\beta_{j}}\left(y_{j}\right), \quad y \in \mathbb{R}^{m}
$$

where

$$
H_{d}(\tau):=\sum_{l=-\infty}^{\infty}\left|\left(\left(\frac{\sin \tau}{\tau+l \pi}\right)^{2}\right)^{(d)}\right| \leq C_{7}(d), \quad \tau \in \mathbb{R}^{1},
$$

by inequalities (2.12) and (2.14) in [11. Therefore, by (3.15), (3.16) and (3.17), we obtain the estimate

$$
I_{2}(x) \leq C_{8} a^{-1}(1+\operatorname{diam}(V))^{N}\|f\|_{L_{\infty}\left(\mathbb{R}^{m}\right)},
$$

where $C_{8}$ is independent of $x, a, f$, and $V$. Combining (3.13) with (3.14) and (3.18), we arrive at (3.12).

Property 3.6. Let $\gamma(a):[1, \infty) \rightarrow(0,1)$ be a function such that $\lim _{a \rightarrow \infty} \gamma(a)=0$. For any $V \subset \mathbb{R}^{m}, q \in(0, \infty]$, and $N \in \mathbb{Z}_{+}^{1}$ there exists a family of numbers $\left\{M_{a}\right\}_{a \in[1, \infty)}$, satisfying the following conditions:

(a) $M_{a} \in(0, a \pi], a \in[1, \infty)$.

(b) $\lim _{a \rightarrow \infty} M_{a}=\infty$.

(c) For every family of functions $\left\{f_{(a)}\right\}_{a \in[1, \infty)}$ such that $f_{(a)} \in B_{(1-\gamma(a)) V} \cap L_{\infty}\left(\mathbb{R}^{m}\right), a \in[1, \infty)$, and $\sup _{a \in[1, \infty)}\left\|f_{(a)}\right\|_{L_{\infty}\left(\mathbb{R}^{m}\right)}<\infty$, the following relation holds true:

$$
\lim _{a \rightarrow \infty}\left\|D_{N}\left(f_{(a)}\right)-D_{N}\left(S_{a}\left(f_{(a)}, \cdot\right)\right)\right\|_{L_{q}\left(Q_{M_{a}}\right)}=0 .
$$


Proof. Let us set $M_{a}:=\min \left\{a^{\delta}, a \pi\right\}$, where $\delta \in\left(0, \varepsilon_{q, N}\right)$ and

$$
\varepsilon_{q, N}:= \begin{cases}2 q /(2 q+m), & N=0, \\ \min \{q / m, 2 q /(2 q+m)\}, & N \in \mathbb{N},\end{cases}
$$

for $q \in(0, \infty]$. Then conditions (a) and (b) are satisfied. Next by Property 3.4 for $N=0$ and by Property 3.5 for $N \in \mathbb{N}$, we obtain for $q \in(0, \infty]$

$$
\begin{aligned}
\| D_{N}\left(f_{(a)}\right) & -D_{N}\left(S_{a}\left(f_{(a)}, \cdot\right)\right) \|_{L_{q}\left(Q_{M_{a}}\right)} \\
& =\left\{\begin{array}{ll}
O\left([(1-\gamma(a)) \operatorname{diam}(V)+1]^{N} M_{a}^{2+m / q} / a^{2}\right), & N=0, \\
O\left([(1-\gamma(a)) \operatorname{diam}(V)+1]^{N} M_{a}^{2+m / q} / a^{2}+M_{a}^{m / q} / a\right), & N \in \mathbb{N},
\end{array}=o(1),\right.
\end{aligned}
$$

as $a \rightarrow \infty$. Hence condition (c) is satisfied as well.

Remark 3.7. In case of $m=1$ and $\gamma(a)=0$, Property 3.1 was established in [18, 13, 14, while Properties 3.2 through 3.6 were proved in [11].

\section{Proofs of Theorems}

Proof of Theorem 1.2. Let $f \in B_{V} \cap L_{p}\left(\mathbb{R}^{m}\right)$, where $p \in(0, \infty]$, and let $c$ be defined by (3.3). Setting $f_{(a)}(x):=f\left((1+c / a)^{-1} x\right), a \geq 1$, we see from Definition 1.1 and Nikolskii-type inequality (1.16) that $f_{(a)} \in B_{(1+c / a)^{-1} V} \cap L_{\infty}\left(\mathbb{R}^{m}\right)$. We can now consider the multivariate Levitan's polynomial $S_{a}\left(f_{(a)}, \cdot\right)$ defined by (3.4) and (3.5). Since $S_{a}\left(f_{(a)}, a \cdot\right) \in \mathcal{T}_{a V}$ by (3.4), we obtain for $0<p \leq q \leq \infty$

$$
\| D_{N}\left(S_{a}\left(f_{(a)}, \cdot\right)\left\|_{L_{q}\left(Q_{a \pi}\right)} \leq P_{p, q, D_{N}, a, V}\right\| S_{a}\left(f_{(a)}, \cdot\right) \|_{L_{p}\left(Q_{a \pi}\right)} .\right.
$$

In addition, we note that $D_{N}(f) \in L_{q}\left(\mathbb{R}^{m}\right)$ by Bernstein-Nikolskii type inequality (1.18). Therefore setting $\gamma(a):=c /(c+a)$, and using Properties 3.6, 3.3, and inequalities (1.1) and (4.1), we obtain

$$
\begin{aligned}
\left\|D_{N}(f)\right\|_{L_{q}\left(\mathbb{R}^{m}\right)} & =\lim _{a \rightarrow \infty}\left\|D_{N}(f)\right\|_{L_{q}\left((1+c / a)^{-1} Q_{M_{a}}\right)} \\
& =\lim _{a \rightarrow \infty}\left\|D_{N}\left(f_{(a)}\right)\right\|_{L_{q}\left(Q_{M_{a}}\right)} \\
& \leq\left(\lim _{a \rightarrow \infty}\left\|D_{N}\left(f_{(a)}\right)-D_{N}\left(S_{a}\left(f_{(a)}, \cdot\right)\right)\right\|_{L_{q}\left(Q_{M_{a}}\right)}^{\tilde{q}}\right. \\
& \left.+\liminf _{a \rightarrow \infty}\left\|D_{N}\left(S_{a}\left(f_{(a)}, \cdot\right)\right)\right\|_{L_{q}\left(Q_{M_{a}}\right)}\right)^{1 / \tilde{q}} \\
& \leq \liminf _{a \rightarrow \infty}\left\|D_{N}\left(S_{a}\left(f_{(a)}, \cdot\right)\right)\right\|_{L_{q}\left(Q_{a \pi}\right)} \\
& \leq \liminf _{a \rightarrow \infty}\left(P_{p, q, D_{N}, a, V}\left\|S_{a}\left(f_{(a)}, \cdot\right)\right\|_{L_{p}\left(Q_{a \pi}\right)}\right) \\
& \leq \liminf _{a \rightarrow \infty}\left(P_{p, q, D_{N}, a, V}\left\|f_{(a)}\right\|_{L_{p}\left(\mathbb{R}^{m}\right)}\right) \\
& =\liminf _{a \rightarrow \infty} P_{p, q, D_{N}, a, V}\|f\|_{L_{p}\left(\mathbb{R}^{m}\right)} .
\end{aligned}
$$

Thus (1.19) is established. 
Proof of Theorem 1.3. We first note that there exists $a_{0} \in(0, \infty)$ such that for $p \in(0, \infty], N \in \mathbb{Z}^{m}$, and $V \subset \mathbb{R}^{m}$, the following crude estimates of $P_{p, \infty, D_{N}, a, V}$ hold true:

$$
C_{9}\left(p, D_{N}, V\right) \leq \inf _{a \in\left[a_{0}, \infty\right)} P_{p, \infty, D_{N}, a, V} \leq \sup _{a \in\left[a_{0}, \infty\right)} P_{p, \infty, D_{N}, a, V} \leq C_{10}\left(p, D_{N}, V\right) .
$$

The left and right inequalities in (4.2) follow from (1.19) and (1.17), respectively.

We will prove the theorem by constructing a nontrivial function $f_{0} \in B_{V} \cap L_{p}\left(\mathbb{R}^{m}\right), p \in(0, \infty]$, such that

$$
E_{p, \infty, D_{N}, V} \geq\left\|D_{N}\left(f_{0}\right)\right\|_{L_{\infty}\left(\mathbb{R}^{m}\right)} /\left\|f_{0}\right\|_{L_{p}\left(\mathbb{R}^{m}\right)} \geq \limsup _{a \rightarrow \infty} P_{p, \infty, D_{N}, a, V}
$$

Then combining (4.3) with (1.19) for $q=\infty$, we see that $\lim _{a \rightarrow \infty} P_{p, \infty, D_{N}, a, V}$ exists and

$$
E_{p, \infty, D_{N}, V}=\lim _{a \rightarrow \infty} P_{p, \infty, D_{N}, a, V}
$$

This proves (1.20). In addition, $f_{0}$ is an extremal function in (4.4); that is, (1.21) is valid.

It remains to construct a function $f_{0}$, satisfying (4.3). Let $T_{a} \in \mathcal{T}_{a V}$ be a polynomial, satisfying the equality

$$
P_{p, \infty, D_{N}, a, V}=a^{-N-m / p}\left\|D_{N}\left(T_{a}\right)\right\|_{L_{\infty}\left(Q_{\pi}\right)} /\left\|T_{a}\right\|_{L_{p}\left(Q_{\pi}\right)}
$$

The existence of an extremal polynomial $T_{a}$ in (4.5) can be proved by the standard compactness argument. Indeed, given $d \in \mathbb{N}$, let $T_{a, d} \in \mathcal{T}_{a V}$ satisfy the following relations:

$$
\left\|T_{a, d}\right\|_{L_{\infty}\left(Q_{\pi}\right)}=1, \quad\left\|D_{N}\left(T_{a, d}\right)\right\|_{L_{\infty}\left(Q_{\pi}\right)}=\left|D_{N}\left(T_{a, d}\right)(0)\right|
$$

and

$$
a^{N+m / p} P_{p, \infty, D_{N}, a, V}<\left\|D_{N}\left(T_{a, d}\right)\right\|_{L_{\infty}\left(Q_{\pi}\right)} /\left\|T_{a, d}\right\|_{L_{p}\left(Q_{\pi}\right)}+1 / d .
$$

Then there exists a nontrivial polynomial $T_{a} \in \mathcal{T}_{a V}$ and a sequence $\left\{d_{k}\right\}_{k=1}^{\infty} \subseteq \mathbb{N}$ such that for any $\alpha \in \mathbb{Z}_{+}^{m}, \lim _{k \rightarrow \infty} D^{\alpha} T_{a, d_{k}}(x)=D^{\alpha} T_{a}(x)$ uniformly on $Q_{\pi}$. Thus (4.5) holds true.

Next setting $U_{a}(x):=T_{a}(x / a)$, we see that $U_{a} \in B_{V} \cap L_{\infty}\left(\mathbb{R}^{m}\right)$. In addition, it follows from (4.5) that

$$
P_{p, \infty, D_{N}, a, V}=\left\|D_{N}\left(U_{a}\right)\right\|_{L_{\infty}\left(Q_{a \pi}\right)} /\left\|U_{a}\right\|_{L_{p}\left(Q_{a \pi}\right)} .
$$

Moreover, we can assume that

$$
\left\|D_{N}\left(U_{a}\right)\right\|_{L_{\infty}\left(Q_{a \pi}\right)}=\left|D_{N}\left(U_{a}\right)(0)\right|=1 .
$$

Note that normalization (4.8) is different compared with (4.6). Normalization (4.6) was used only for the proof of the existence of extremal polynomials $T_{a}$ in (4.5). 
Then we obtain from (4.7), (4.8), and (4.2)

$$
\begin{aligned}
& \sup _{a \in\left[a_{0}, \infty\right)}\left\|U_{a}\right\|_{L_{\infty}\left(\mathbb{R}^{m}\right)} \leq \sup _{a \in\left[a_{0}, \infty\right)}\left(P_{p, \infty, D_{0}, a, V}\left\|U_{a}\right\|_{L_{p}\left(Q_{a \pi}\right)}\right) \\
= & \sup _{a \in\left[a_{0}, \infty\right)}\left(P_{p, \infty, D_{0}, a, V} / P_{p, \infty, D_{N}, a, V}\right) \leq C_{10}\left(p, D_{0}, V\right) / C_{9}\left(p, D_{N}, V\right) .
\end{aligned}
$$

Let $\left\{a_{s}\right\}_{s=1}^{\infty} \subset\left[a_{0}, \infty\right)$ be a sequence such that

$$
\limsup _{a \rightarrow \infty} P_{p, \infty, D_{N}, a, V}=\lim _{s \rightarrow \infty} P_{p, \infty, D_{N}, a_{s}, V}
$$

Using now the compactness theorem of Lemma 2.3 for the sequence $\left\{U_{a_{s}}\right\}_{s=1}^{\infty}$ of functions from $B_{V} \cap L_{\infty}\left(\mathbb{R}^{m}\right)$ uniformly bounded by (4.9), we see that there exist a function $f_{0} \in B_{V} \cap L_{\infty}\left(\mathbb{R}^{m}\right)$ and a subsequence $\left\{a_{s_{r}}\right\}_{r=1}^{\infty}$ such that

$$
\lim _{r \rightarrow \infty} D^{\alpha} U_{a_{s_{r}}}(x)=D^{\alpha} f_{0}(x), \quad \alpha \in \mathbb{Z}_{+}^{m}
$$

uniformly on any cube $Q_{A} \subset \mathbb{R}^{m}, A>0$. Moreover, by (4.8) and (4.11),

$$
\left\|D_{N}\left(f_{0}\right)\right\|_{L_{\infty}\left(\mathbb{R}^{m}\right)}=\left|D_{N}\left(f_{0}\right)(0)\right|=1
$$

In addition, using (1.1), (4.11), (4.7), and (4.8), we obtain for any cube $Q_{A} \subset \mathbb{R}^{m}, A>0$,

$$
\begin{aligned}
& \left\|f_{0}\right\|_{L_{p}\left(Q_{A}\right)}^{\tilde{p}} \leq \lim _{r \rightarrow \infty}\left(\left\|f_{0}-U_{a_{s_{r}}}\right\|_{L_{p}\left(Q_{A}\right)}^{\tilde{p}}+\left\|U_{a_{s_{r}}}\right\|_{L_{p}\left(Q_{A}\right)}^{\tilde{p}}\right) \\
& \leq \lim _{r \rightarrow \infty}\left\|U_{a_{s_{r}}}\right\|_{L_{p}\left(Q_{a_{s_{r}} \pi}\right)}^{\tilde{p}}=1 / \lim _{r \rightarrow \infty} P_{p, \infty, D_{N}, a_{s_{r}}, V}^{\tilde{p}} .
\end{aligned}
$$

Next using (4.13) and (4.2), we see that

$$
\left\|f_{0}\right\|_{L_{p}\left(Q_{A}\right)} \leq 1 / C_{9}\left(p, D_{N}, V\right)
$$

Therefore, $f_{0}$ is a nontrivial function from $B_{V} \cap L_{p}\left(\mathbb{R}^{m}\right)$, by (4.12) and (4.14). Thus for any cube $Q_{A} \subset \mathbb{R}^{m}, A>0$, we obtain from (4.10), (4.7), (4.11), and (4.12)

$$
\begin{aligned}
& \limsup _{a \rightarrow \infty} P_{p, \infty, D_{N}, a, V}=\lim _{r \rightarrow \infty}\left(\left\|U_{a_{s_{r}}}\right\|_{L_{p}\left(Q_{a_{s_{r}}}\right)}\right)^{-1} \\
& \leq \lim _{r \rightarrow \infty}\left(\left\|U_{a_{s_{r}}}\right\|_{L_{p}\left(Q_{A}\right)}\right)^{-1}=\left\|D_{N}\left(f_{0}\right)\right\|_{L_{\infty}\left(\mathbb{R}^{m}\right)} /\left\|f_{0}\right\|_{L_{p}\left(Q_{A}\right)} .
\end{aligned}
$$

Finally, letting $A \rightarrow \infty$ in (4.15), we arrive at (4.3). 


\section{REFERENCES}

[1] N. I. Akhiezer, Lectures on the Theory of Approximation. 2nd ed., Nauka, Moscow, 1965 (in Russian).

[2] V. V. Arestov, On integral inequalities for trigonometric polynomials and their derivatives, Izv. Akad. Nauk SSSR, Ser. Mat. 45 (1981), 3-22 (in Russian); English transl. in Math. USSR-Izv. 18 (1982), 1-17.

[3] S. N. Bernstein, On entire functions of finite degree of several variables, Dokl. Akad. Nauk SSSR 60 (1948), 949-952 (in Russian).

[4] R. P. Boas, Entire Functions, Academic Press, New York, 1954.

[5] F. Dai, D. Gorbachev, and S. Tikhonov, Nikolskii constants for polynomials on the unit sphere, J. Anal. Math. (2019), in press; arXiv:1708.09837v1 (2017).

[6] Z. Ditzian, A. Prymak, Nikolskii inequalities for Lorentz spaces, Rocky Mountain Journal of Mathematics 40(1) (2010), 209-223.

[7] M. I. Ganzburg, The theorems of Jackson and Bernstein in $\mathbb{R}^{m}$, Uspekhi Mat. Nauk 34(1) (1979), 225-226 (in Russian); English transl. in Russian Math. Surveys 34(1) (1979), 221-222.

[8] M. I. Ganzburg, Multidimensional Bernstein-type inequalities, Ukrainskii Matematicheskii Zhurnal 34 (1982), 749-753 (in Russian); English transl. in Ukranian Math. J. 34 (1982), 607-610.

[9] M. I. Ganzburg, Multidimensional polynomials of Levitan, in The Approximation of the Functions and Summation of the Series, pp. 112-117, Dniepropetrovsk State University, Dniepropetrovsk, 1990.

[10] M. I. Ganzburg, Polynomial inequalities on measurable sets and their applications, Constr. Approx. 17 (2001), 275-306.

[11] M. I. Ganzburg, S. Yu. Tikhonov, On sharp constants in Bernstein-Nikolskii inequalities, Constr. Approx. 45 (2017), 449-466.

[12] D. V. Gorbachev, I. A. Martyanov, On the interrelation of the Nikol'skii constant for trigonometric polynomials and entire functions of exponential type, Chebyshevskii Sb. 19 (2018), no. 2, 80-89 (in Russian).

[13] L. Hörmander, A new proof and a generalization of an inequality of Bohr, Math. Scand. 2 (1954), 33-45.

[14] L. Hörmander, Some inequalities for functions of exponential type, Math. Scand. 3 (1955), 21-27.

[15] A. I. Kamzolov, On Riesz's interpolation formula and Bernshtein's inequality for functions on homogeneous spaces, Mat. Zametki 15 (1974), 967-978 (in Russian); English transl. in Math. Notes 15 (1974), 576-582.

[16] E. Levin, D. Lubinsky, $L_{p}$ Christoffel functions, $L_{p}$ universality, and Paley-Wiener spaces, J. D'Analyse Math. 125 (2015), 243-283.

[17] E. Levin, D. Lubinsky, Asymptotic behavior of Nikolskii constants for polynomials on the unit circle, Comput. Methods Funct. Theory 15 (2015), 459-468.

[18] B. M. Lewitan, Über eine Verallgemeinerung der Ungleichungen von S. Bernstein und H. Bohr, Dokl. Akad. Nauk SSSR 15 (1937), 169-172.

[19] R. J. Nessel, G. Wilmes, Nikolskii-type inequalities for trigonometric polynomials and entire functions of exponential type, J. Austral. Math. Soc., Ser. A 25 (1978), 7-18.

[20] S. M. Nikolskii, Inequalities for entire functions of finite degree and their application to the theory of differentiable functions of several variables, Trudy Mat. Inst. Steklov 38 (1951), 244-278 (in Russian); English transl. in Amer. Math. Soc. Transl. Ser. 2, 80 (1969), 1-38. 
[21] S. M. Nikolskii, Approximation of Functions of Several Variables and Imbedding Theorems, Nauka, Moscow, 1969 (in Russian); English edition: Die Grundlehren der Mathematischen Wissenschaften, Band 205, Springer-Verlag, New York-Heidelberg, 1975.

[22] Q. I. Rahman, G. Schmeisser, $L^{p}$ inequalities for entire functions of exponential type, Trans. Amer. Math. Soc. 320 (1990), 91-103.

[23] E. Stein, G. Weiss, Introduction to Fourier Analysis on Euclidean Spaces, Princeton Univ. Press, Princeton, 1971.

[24] A. F. Timan, Theory of Approximation of Functions of a Real Variable, Pergamon Press, New York, 1963.

Department of Mathematics, Hampton University, Hampton, VA 23668, USA

E-mail address: michael.ganzburg@hamptonu.edu 